Vietnam Journal of Mechanics, VAST, Vol.41, No. 4 (2019), pp. 349-361

DOI: https://doi.org/10.15625/0866-7136/14566

\title{
EXACT RECEPTANCE FUNCTION AND RECEPTANCE CURVATURE OF A CLAMPED-CLAMPED CONTINUOUS CRACKED BEAM
}

\author{
Nguyen Viet Khoa*, Cao Van Mai, Dao Thi Bich Thao \\ Institute of Mechanics, VAST, 18 Hoang Quoc Viet, Hanoi, Vietnam \\ *E-mail: nvkhoa@imech.vast.vn
}

Received: 21 October 2019 / Published online: 24 December 2019

\begin{abstract}
The receptance function has been studied and applied widely since it interrelates the harmonic excitation and the response of a structure in the frequency domain. This paper presents the derivation of the exact receptance function of continuous cracked beams and its application for crack detection. The receptance curvature is defined as the second derivative of the receptance. The influence of the crack on the receptance and receptance curvature is investigated. It is concluded that when there are cracks the receptance curvature has sharp changes at the crack positions. This can be applied for the crack detection purpose. In this paper, the numerical simulations are provided.

Keywords: receptance, curvature of receptance, frequency response function, crack, crack detection.
\end{abstract}

\section{INTRODUCTION}

The receptance method which was first introduced by Bishop and Johnson [1] has been applied wildly in mechanical system and structural dynamics. Yang [2] presented the exact receptances of non-proportionally damped dynamic systems. Based on a decomposition of the damping matrix, an iteration procedure is developed which does not require matrix inversion. Mottershead [3] investigated the measured zeros form frequency response functions and its application to model assessment and updating. Gurgoze [4] was concerned with receptance matrices of viscously damped systems subject to several constraint equations. The frequency response matrix of the constrained system was established in terms of the frequency response matrix of the unconstrained system and the coefficient vectors of the constraint equations. Karakas and Gürgöze [5] presented a formulation of the receptance matrix of non-proportionally damped dynamic systems. The receptance matrix was obtained directly without using the iterations as presented in [1]. Albertelli et al. [6] proposed a method using receptance coupling substructure method to improve chatter free cutting conditions prediction. Recently, Muscolino and Santoro [7] presented the explicit frequency response function of beams with

(C) 2019 Vietnam Academy of Science and Technology 
cracks of uncertain depths in order to evaluate the main statistics as well as the upper and lower bounds of the response.

The cracks can influence significantly the dynamic characteristics of structures such as natural frequencies, mode shapes, etc. These dynamic characteristics have been investigated and applied wildly for crack detection of structures. Lee and Chung [8] studied the change in natural frequencies of beams caused by a crack. Zheng and Kessissoglou [9] investigated the relationship between natural frequency of a cracked beam to the depth and location of the crack. The results in these papers showed that the natural frequency of the cracked beam decreases as the crack depth increases. Gudmundson [10] investigated the influence of cracks on the natural frequencies of slender structures using a flexibility matrix approach. Thalapil and Maiti [11] revealed the change in natural frequencies caused by longitudinal cracks for crack detection. Khoa [12] proposed a method for monitoring a sudden crack of a beam-like bridge appeared during earthquake excitation based on the instantaneous frequency extracted from wavelet power spectrum. Some authors presented methods to calculate and apply the mode shape of cracked structures for crack detection purposes. Caddemi and Calio $[13,14]$ presented the exact closed-form solution for the mode shapes of the Euler-Bernoulli beam with multiple open cracks. Lien et al. [15] presented a mode shape analysis of multiple cracked functionally graded beamlike structures by using dynamic stiffness method for crack detection purpose. One of authors of this paper applied 3D finite elements to investigate the change in mode shapes at the crack positions [16]. The results showed that the sharp changes in mode shapes at the crack positions can be applied for detecting small cracks.

In most of the previous works the receptance of beams was derived discretely. In this paper the exact formulas of receptance function and receptance curvature of a cracked beam will be established. The receptance curvature is defined as the second derivative of the receptance with respect to the coordinate of beam. The effect of the crack on the receptance curvature of cracked beams is investigated. The result showed that the receptance curvature has significant changes at crack positions. This result can be used for crack detection. The numerical simulations are provided in this paper.

\section{DERIVATION OF THE RECEPTANCE FUNCTION OF A CRACKED BEAM}

\subsection{Intact beam}

In this work, the undamped Euler-Bernoulli beam is considered. The forced vibration equation of undamped beam can be written as follows

$$
\frac{\partial^{2}}{\partial \xi^{2}} E I(\xi) \frac{\partial^{2} v(\xi, t)}{\partial \xi^{2}}+L^{4} m(\xi) \frac{\partial^{2} v(\xi, t)}{\partial t^{2}}=L^{4} P(\xi, t),
$$

where $\xi=\frac{x}{L}$ is the non-dimensional coordinate. The solution of Eq. (1) can be found in the form

$$
v(\xi, t)=\sum_{k=1}^{\infty} \phi_{k}(\xi) Y_{k}(t)
$$


where $\phi_{k}(x)$ is the $k^{\text {th }}$ mode shape of the beam, $Y_{k}(t)$ is the time-dependent amplitude which is referred to as generalized coordinate. Substituting Eq. (2) into Eq. (1), multiplying both sides of the equation with $\phi_{n}(x)$ integrating and applying orthogonality conditions of beam gives

$$
\ddot{Y}_{n}(t) \int_{0}^{1} m(\xi) \phi_{n}^{2}(\xi) \mathrm{d} \xi+Y_{n}(t) \omega_{n}^{2} \int_{0}^{1} m(\xi) \phi_{n}^{2}(\xi) \mathrm{d} \xi=\int_{0}^{1} \phi_{n}(\xi) P(\xi, t) \mathrm{d} \xi .
$$

If the force $P$ excites at a point $\xi=\hat{\xi}$, Eq. (3) becomes

$$
\ddot{Y}_{n}(t) \int_{0}^{1} m(\xi) \phi_{n}^{2}(\xi) \mathrm{d} \xi+Y_{n}(t) \omega_{n}^{2} \int_{0}^{1} m(\xi) \phi_{n}^{2}(\xi) \mathrm{d} \xi=\phi_{n}(\hat{\xi}) P(\hat{\xi}, t) .
$$

If the force is harmonic $P(\hat{\xi}, t)=\hat{P} \sin \omega t$ exciting at the point $\hat{\xi}$, then we have $Y_{n}(t)=$ $\bar{Y}_{n} \sin \omega t$, where $\bar{Y}_{n}$ is the amplitude. Multiply both sides of Eq. (4) by $\phi_{n}(\xi)$, yields

$$
\left(\omega_{n}^{2}-\omega^{2}\right) \bar{Y}_{n} \phi_{n}(\xi) \int_{0}^{1} m(\xi) \phi_{n}^{2}(\xi) \mathrm{d} \xi=\phi_{n}(\xi) \phi_{n}(\hat{\xi}) \hat{P} .
$$

From Eq. (5) the following formula is derived

$$
\sum_{n=1}^{\infty} \frac{\bar{Y}_{n}(\omega) \phi_{n}(\xi)}{P}=\sum_{n=1}^{\infty} \frac{\phi_{n}(\xi) \phi_{n}(\hat{\xi})}{\left(\omega_{n}^{2}-\omega^{2}\right) \int_{0}^{1} m(\xi) \phi_{n}^{2}(\xi) \mathrm{d} \xi} .
$$

Therefore, the receptance at $\xi$ due to the force at $\hat{\xi}$ is

$$
\alpha_{\xi \hat{\xi}}(\omega)=\sum_{n=1}^{\infty} \frac{1}{\omega_{n}^{2}-\omega^{2}} \frac{\phi_{n}(\xi) \phi_{n}(\hat{\zeta})}{\int_{0}^{1} m(\xi) \phi_{n}^{2}(\xi) \mathrm{d} \xi} .
$$

\subsection{Cracked beam}

Although in general the change in mode shapes caused by the crack at the crack position is small when the crack depth is small, the curvature of the mode shape at the crack position can be significant since the mode shape is changed sharply at the crack position. In this paper define the "receptance curvature" as the second derivative of the receptance function with respect to $\xi$ variable as follows

$$
\frac{\partial^{2} \alpha_{\tilde{\xi} \hat{\xi}}(\omega)}{\partial \xi^{2}}=\sum_{n=1}^{\infty} \frac{1}{\omega_{n}^{2}-\omega^{2}} \frac{\phi_{n}(\hat{\zeta})}{m \int_{0}^{1} \phi_{n}^{2}(\xi) \mathrm{d} \xi} \frac{\mathrm{d}^{2} \phi_{n}(\xi)}{\mathrm{d} \xi^{2}} .
$$


Here we consider the elementary case with $m(x)$ set equal to constant $m$. The exact closed form of the mode shape of a clamped-clamped beam with $n$ cracks is adopted from [13] as follows

$$
\begin{aligned}
\phi_{k}(\xi)= & C_{1}\left\{\frac{1}{2 \alpha_{k}} \sum_{i=1}^{n} \lambda_{i} \mu_{i}\left[\sin \alpha_{k}\left(\xi-\xi_{0 i}\right)+\sinh \alpha_{k}\left(\xi-\xi_{0 i}\right)\right] U\left(\xi-\xi_{0 i}\right)+\sin \alpha_{k} \xi\right\} \\
& +\left\{\frac{1}{2 \alpha_{k}} \sum_{i=1}^{n} \lambda_{i} v_{i}\left[\sin \alpha_{k}\left(\xi-\xi_{0 i}\right)+\sinh \alpha_{k}\left(\xi-\xi_{0 i}\right)\right] U\left(\xi-\xi_{0 i}\right)+\cos \alpha_{k} \xi\right\} \\
& -C_{1}\left\{\frac{1}{2 \alpha_{k}} \sum_{i=1}^{n} \lambda_{j} \zeta_{i}\left[\sin \alpha_{k}\left(\xi-\xi_{0 i}\right)+\sinh \alpha_{k}\left(\xi-\xi_{s i}\right)\right] U\left(\xi-\xi_{0 i}\right)+\sinh \alpha_{k} \xi\right\} \\
& -\left\{\frac{1}{2 \alpha_{k}} \sum_{i=1}^{n} \lambda_{i} \eta_{i}\left[\sin \alpha_{k}\left(\xi-\xi_{0 i}\right)+\sinh \alpha_{k}\left(\xi-\xi_{0 i}\right)\right] U\left(\xi-\xi_{0 i}\right)+\cosh \alpha_{k} \xi\right\},
\end{aligned}
$$

where

$$
\begin{aligned}
& C_{1}=-\frac{\frac{1}{2 \alpha_{k}} \sum_{i=1}^{n} \lambda_{i}\left(v_{i}-\eta_{i}\right)\left[\sin \alpha_{k}\left(1-\xi_{o i}\right)+\sinh \alpha_{k}\left(1-\xi_{o i}\right)\right]+\cos \alpha_{k}-\cosh \alpha_{k}}{\frac{1}{2 \alpha_{k}} \sum_{i=1}^{n} \lambda_{i}\left(\mu_{i}-\zeta_{i}\right)\left[\sin \alpha_{k}\left(1-\xi_{o i}\right)+\sinh \alpha_{k}\left(1-\xi_{o i}\right)\right]+\sin \alpha_{k}-\sinh \alpha_{k}}, \\
& C_{2}=-C_{4}=1, \quad C_{3}=-C_{1},
\end{aligned}
$$

$\alpha_{k}$ is the dimensionless frequency parameter $\alpha_{k}^{4}=\frac{\omega_{k}^{2} m L^{4}}{E I} ; \xi_{0 i}$ is the position of the $i^{t h}$ crack, where $0<\xi_{01}<\xi_{02}<\ldots<\xi_{0 n}<1$; $U$ is Heaviside function. The terms $\mu_{i}, v_{i}, \zeta_{i}$, $\eta_{i}$ are calculated recurrently by the following equations

$$
\begin{aligned}
& \mu_{j}=\frac{\alpha}{2} \sum_{i=1}^{j-1} \lambda_{i} \mu_{i}\left[-\sin \alpha\left(\xi_{0 j}-\xi_{0 i}\right)+\sinh \alpha_{k}\left(\xi_{0 j}-\xi_{0 i}\right)\right]-\alpha^{2} \sin \alpha \xi_{0 j}, \\
& v_{j}=\frac{\alpha}{2} \sum_{i=1}^{j-1} \lambda_{i} v_{i}\left[-\sin \alpha\left(\xi_{0 j}-\xi_{0 i}\right)+\sinh \alpha_{k}\left(\xi_{0 j}-\xi_{0 i}\right)\right]-\alpha^{2} \cos \alpha \xi_{0 j}, \\
& \zeta_{j}=\frac{\alpha}{2} \sum_{i=1}^{j-1} \lambda_{i} s_{i}\left[-\sin \alpha\left(\xi_{0 j}-\xi_{0 i}\right)+\sinh \alpha_{k}\left(\xi_{0 j}-\xi_{0 i}\right)\right]+\alpha^{2} \sinh \alpha \xi_{0 j}, \\
& \eta_{j}=\frac{\alpha}{2} \sum_{i=1}^{j-1} \lambda_{i} \eta_{i}\left[-\sin \alpha\left(\xi_{0 j}-\xi_{0 i}\right)+\sinh \alpha_{k}\left(\xi_{0 j}-\xi_{0 i}\right)\right]+\alpha^{2} \cosh \alpha \xi_{0 j}
\end{aligned}
$$

In order to derive the exact formulas of receptance and curvature receptance as presented in Eqs. (9) and (10), the second derivative of the mode shape and the integral of the square of the mode shape need to be calculated.

For simplicity, the operator $S\left(\alpha_{k}, \xi\right)=\sin \alpha_{k}\left(\xi-\xi_{0 i}\right)+\sinh \alpha_{k}\left(\xi-\xi_{0 i}\right)$ is presented. The second derivative of the mode shape with respected to $\xi$ can be obtained as follows

$$
(S U)^{\prime \prime}=S^{\prime \prime} U+S U^{\prime \prime}+2 S^{\prime} U^{\prime} \text {. }
$$


Applying the following properties of Heaviside function and Dirac delta function [17]

$$
U^{\prime}(\xi)=\delta(\xi), f(\xi) \delta^{\prime}(\xi)=-f^{\prime}(\xi) \delta(\xi),
$$

yields

$$
\begin{aligned}
(S U)^{\prime \prime}= & \alpha_{k}^{2}\left[\sinh \alpha_{k}\left(\xi-\xi_{0 i}\right)-\sin \alpha_{k}\left(\xi-\xi_{0 i}\right)\right] U\left(\xi-\xi_{0 i}\right) \\
& +\alpha_{k}\left[\cos \alpha_{k}\left(\xi-\xi_{0 i}\right)+\cosh \alpha_{k}\left(\xi-\xi_{0 i}\right)\right] \delta\left(\xi-\xi_{0 i}\right)
\end{aligned}
$$

From Eq. (9) and Eq. (14), the second derivative of the mode shape can be derived as follows

$$
\begin{aligned}
\phi_{k}^{\prime \prime}(\xi)= & C_{1}\left\{\frac { 1 } { 2 } \sum _ { i = 1 } ^ { n } \lambda _ { i } \mu _ { i } \left[\alpha_{k}\left(\sinh \alpha_{k}\left(\xi-\xi_{0 i}\right)-\sin \alpha_{k}\left(\xi-\xi_{0 i}\right)\right) U\left(\xi-\xi_{0 i}\right)\right.\right. \\
& \left.\left.+\left(\cos \alpha_{k}\left(\xi-\xi_{0 i}\right)+\cosh \alpha_{k}\left(\xi-\xi_{0 i}\right)\right) \delta\left(\xi-\xi_{0 i}\right)\right]-\alpha_{k}^{2} \sin \alpha_{k} \xi\right\} \\
& +\left\{\frac { 1 } { 2 } \sum _ { i = 1 } ^ { n } \lambda _ { i } v _ { i } \left[\alpha_{k}\left(\sinh \alpha_{k}\left(\xi-\xi_{0 i}\right)-\sin \alpha_{k}\left(\xi-\xi_{0 i}\right)\right) U\left(\xi-\xi_{0 i}\right)\right.\right. \\
& \left.\left.+\left(\cos \alpha_{k}\left(\xi-\xi_{0 i}\right)+\cosh \alpha_{k}\left(\xi-\xi_{0 i}\right)\right) \delta\left(\xi-\xi_{0 i}\right)\right]-\alpha_{k}^{2} \cos \alpha_{k} \xi\right\} \\
& -C_{1}\left\{\frac { 1 } { 2 } \sum _ { i = 1 } ^ { n } \lambda _ { i } \zeta _ { i } \left[\alpha_{k}\left(\sinh \alpha_{k}\left(\xi-\xi_{0 i}\right)-\sin \alpha_{k}\left(\xi-\xi_{0 i}\right)\right) U\left(\xi-\xi_{0 i}\right)\right.\right. \\
& \left.\left.+\left(\cos \alpha_{k}\left(\xi-\xi_{0 i}\right)+\cosh \alpha_{k}\left(\xi-\xi_{0 i}\right)\right) \delta\left(\xi-\xi_{0 i}\right)\right]+\alpha_{k}^{2} \sinh \alpha_{k} \xi\right\} \\
& -\left\{\frac { 1 } { 2 } \sum _ { i = 1 } ^ { n } \lambda _ { i } \eta _ { i } \left[\alpha_{k}\left(\sinh \alpha_{k}\left(\xi-\xi_{0 i}\right)-\sin \alpha_{k}\left(\xi-\xi_{0 i}\right)\right) U\left(\xi-\xi_{0 i}\right)\right.\right. \\
& \left.\left.+\left(\cos \alpha_{k}\left(\xi-\xi_{0 i}\right)+\cosh \alpha_{k}\left(\xi-\xi_{0 i}\right)\right) \delta\left(\xi-\xi_{0 i}\right)\right]+\alpha_{k}^{2} \cosh \alpha_{k} \xi\right\}
\end{aligned}
$$

Applying the property of Heaviside function, we have

$$
\int_{0}^{1} f(\xi) U\left(\xi-\xi_{0 i}\right) \mathrm{d} \xi=\int_{\xi_{0 i}}^{1} f(\xi) \mathrm{d} \xi=F(1)-F\left(\xi_{0 i}\right),
$$

where $F$ is the antiderivative function of $f$.

It is noted that

$$
U\left(\xi-\xi_{0 i}\right) U\left(\xi-\xi_{0 j}\right)=\left[\begin{array}{ll}
U\left(\xi-\xi_{0 i}\right), & i \geq j \\
U\left(\xi-\xi_{0 j}\right), & i<j
\end{array}\right.
$$

From Eqs. (16) and (17) we have

$$
\begin{aligned}
& \int_{0}^{1} f(\xi) U\left(\xi-\xi_{0 i}\right) U\left(\xi-\xi_{0 j}\right) \mathrm{d} \xi= \\
& F(1)-F\left(\xi_{0 i}\right) U\left(\xi_{0 i}-\xi_{0 j}\right)-F\left(\xi_{0 j}\right) U\left(\xi_{0 j}-\xi_{0 i}\right)+F\left(\xi_{0 i}\right) \delta_{i j}
\end{aligned}
$$

where $\delta_{i j}$ is the Kronecker delta. 
Analytical calculations show that the term $F\left(\xi_{0 i}\right) \delta_{i j}$ in Eq. (18) vanishes. From Eqs. (9) to (18), the following equation is obtained

$$
\begin{aligned}
& \int_{0}^{1} \phi_{k}^{2}(\xi) \mathrm{d} \xi=\frac{1}{8 \alpha_{k}^{2}} \sum_{i=1}^{n} \sum_{j=1}^{n} \lambda_{i} \lambda_{j} A_{1} \times\left\{\cos \alpha_{k}\left(\xi_{0 i}-\xi_{0 j}\right)-\frac{1}{2 \alpha_{k}} \sin \alpha_{k}\left(2-\xi_{0 i}-\xi_{0 j}\right)\right. \\
& -\cosh \alpha_{k}\left(\xi_{0 i}-\xi_{0 j}\right)+\frac{1}{\alpha_{k}} \sinh \alpha_{k}\left(1-\xi_{0 i}\right) \cosh \alpha_{k}\left(1-\xi_{0 j}\right) \\
& -\frac{1}{\alpha_{k}} \cos \alpha_{k}\left(1-\xi_{0 i}\right) \sinh \alpha_{k}\left(1-\xi_{0 j}\right)+\frac{1}{\alpha_{k}} \sin \alpha_{k}\left(1-\xi_{0 i}\right) \cosh \alpha_{k}\left(1-\xi_{0 j}\right) \\
& -\frac{1}{\alpha_{k}} \cos \alpha_{k}\left(1-\xi_{0 j}\right) \sinh \alpha_{k}\left(1-\xi_{0 i}\right)+\frac{1}{\alpha_{k}} \sin \alpha_{k}\left(1-\xi_{0 j}\right) \cosh \alpha_{k}\left(1-\xi_{0 i}\right) \\
& -\left[\xi_{0 i} \cos \alpha_{k}\left(\xi_{0 i}-\xi_{0 j}\right)+\frac{1}{2 \alpha_{k}} \sin \alpha_{k}\left(\xi_{0 i}-\xi_{0 j}\right)-\xi_{0 i} \cosh \alpha_{k}\left(\xi_{0 i}-\xi_{0 j}\right)\right. \\
& \left.-\frac{1}{\alpha_{k}} \sinh \alpha_{k}\left(\xi_{0 i}-\xi_{0 j}\right)\right] U\left(\xi_{0 i}-\xi_{0 j}\right) \\
& \left.-\left[\xi_{0 j} \cos \alpha_{k}\left(\xi_{0 j}-\xi_{0 i}\right)+\frac{1}{2 \alpha_{k}} \sin \alpha_{k}\left(\xi_{0 i}-\xi_{0 i}\right)-\xi_{0 j} \cosh \alpha_{k}\left(\xi_{0 j}-\xi_{0 i}\right)\right] U\left(\xi_{0 j}-\xi_{0 i}\right)\right\} \\
& +\frac{1}{2 \alpha_{k} \sum_{i=1}^{n} \lambda_{i} A_{2}}\left[\left(1-\xi_{0 i}\right) \cos \alpha_{k} \xi_{0 i}-\frac{1}{2 \alpha_{k}} \sin \alpha_{k} \xi_{0 i}-\frac{1}{2 \alpha_{k}} \sin \alpha_{k}\left(2-\xi_{0 i}\right)\right. \\
& \left.+\frac{1}{\alpha_{k}} \sin \alpha_{k} \cosh \alpha_{k}\left(1-\xi_{0 i}\right)-\frac{1}{\alpha_{k}} \cos \alpha_{k} \sinh \alpha_{k}\left(1-\xi_{0 i}\right)\right] \\
& +\frac{1}{2 \alpha_{k}} \sum_{i=1}^{n} \lambda_{i} A_{3}\left[\left(\xi_{0 i}-1\right) \sin \alpha_{k} \xi_{0 i}-\frac{1}{2 \alpha_{k}} \cos \alpha_{k} \xi_{0 i}-\frac{1}{2 \alpha_{k}} \cos \alpha_{k}\left(2-\xi_{0 i}\right)\right. \\
& \left.+\frac{1}{\alpha_{k}} \sin \alpha_{k} \sinh \alpha_{k}\left(1-\xi_{0 i}\right)+\frac{1}{\alpha_{k}} \cos \alpha_{k} \cosh \alpha_{k}\left(1-\xi_{0 i}\right)\right] \\
& +\frac{1}{2 \alpha_{k}} \sum_{i=1}^{n} \lambda_{i} A_{4}\left[\left(\xi_{0 i}-1\right) \cosh \alpha_{k} \xi_{0 i}+\frac{1}{2 \alpha_{k}} \sinh \alpha_{k} \xi_{0 i}+\frac{1}{2 \alpha_{k}} \sinh \alpha_{k}\left(2-\xi_{0 i}\right)\right. \\
& \left.+\frac{1}{\alpha_{k}} \sin \alpha_{k}\left(1-\xi_{0 i}\right) \cosh \alpha_{k}-\frac{1}{\alpha_{k}} \cos \alpha_{k}\left(1-\xi_{0 i}\right) \sinh \alpha_{k}\right] \\
& +\frac{1}{2 \alpha_{k}} \sum_{i=1}^{n} \lambda_{i} A_{5}\left[\left(\xi_{0 i}-1\right) \sinh \alpha_{k} \xi_{0 i}+\frac{1}{2 \alpha_{k}} \cosh \alpha_{k} \xi_{0 i}+\frac{1}{2 a_{k}} \cosh \alpha_{k}\left(2-\xi_{0 i}\right)\right. \\
& \left.+\frac{1}{\alpha_{k}} \sin \alpha_{k}\left(1-\xi_{0 i}\right) \sinh \alpha_{k}-\frac{1}{\alpha_{k}} \cos \alpha_{k}\left(1-\xi_{0 i}\right) \cosh \alpha_{k}\right] \\
& +\left[1+\frac{1-C_{1}^{2}}{4 \alpha_{k}} \sin 2 \alpha_{k}+\frac{C_{1}^{2}+1}{4 \alpha_{k}} \sinh 2 \alpha_{k}+\frac{C_{1}}{\alpha_{k}} \sin ^{2} \alpha_{k}+\frac{C_{1}}{\alpha_{k}} \sinh ^{2} \alpha_{k}\right. \\
& \left.-\frac{C_{1}^{2}+1}{\alpha_{k}} \sin \alpha_{k} \cosh \alpha_{k}+\frac{C_{1}^{2}-1}{\alpha_{k}} \cos \alpha_{k} \sinh \alpha_{k}-\frac{2 C_{1}}{\alpha_{k}} \sin \alpha_{k} \sinh \alpha_{k}\right] \\
& +\left[C_{1} C_{2} \frac{\sin ^{2} \alpha_{k}}{\alpha_{k}}+C_{3} C_{4} \frac{\sinh ^{2} \alpha_{k}}{\alpha_{k}}+\left(C_{2}^{2}-C_{1}^{2}\right) \frac{\sin 2 \alpha_{k}}{4 \alpha_{k}}+\left(C_{3}^{2}+C_{4}^{2}\right) \frac{\sinh 2 \alpha_{k}}{4 \alpha_{k}}\right. \\
& +\left(C_{1} C_{3}+C_{2} C_{4}\right) \frac{\sin \alpha_{k} \cosh \alpha_{k}}{\alpha_{k}}+\left(C_{2} C_{4}-C_{1} C_{3}\right) \frac{\cos \alpha_{k} \sinh \alpha_{k}}{\alpha_{k}}+\left(C_{1} C_{4}+C_{2} C_{3}\right) \frac{\sin \alpha_{k} \sinh \alpha_{k}}{\alpha_{k}} \\
& \left.+\left(C_{2} C_{3}-C_{1} C_{4}\right) \frac{\cos \alpha_{k} \cosh \alpha_{k}}{\alpha_{k}}+\frac{1}{2}\left(C_{1}^{2}+C_{2}^{2}-C_{3}^{2}+C_{4}^{2}\right)+\frac{1}{\alpha_{k}}\left(C_{1} C_{4}-C_{2} C_{3}\right)\right] \text {, }
\end{aligned}
$$


where

$$
\begin{aligned}
& A_{1}=C_{1}^{2} \mu_{i} \mu_{j}+v_{i} v_{j}+C_{1}^{2} \zeta_{i} \zeta_{j}+\eta_{i} \eta_{j}+2 C_{1} \mu_{i} v_{j}-2 C_{1}^{2} \mu_{i} \zeta_{j}-2 C_{1} \mu_{i} \eta_{j}-2 C_{1} v_{i} \zeta_{j}-2 v_{i} \eta_{j}+2 C_{1} \zeta_{i} \eta_{j}, \\
& A_{2}=C_{1}^{2} \mu_{i}+C_{1} v_{i}-C_{1}^{2} \zeta_{i}-C_{1} \eta_{i}, \quad A_{3}=C_{1} \mu_{i}+v_{i}-C_{1} \zeta_{i}-\eta_{i}, \\
& A_{4}=-C_{1}^{2} \mu_{i}-C_{1} v_{i}+C_{1}^{2} \zeta_{i}+C_{1} \eta_{i}, \quad A_{5}=-C_{1} \mu_{i}-v_{i}+C_{1} \zeta_{i}+\eta_{i} .
\end{aligned}
$$

Substituting Eqs. (15) to (20) into Eqs. (7) and (8) the exact receptance and curvature receptance of the simply supported beam will be obtained.

Formulas of the receptance and receptance curvature of beam with other general boundary conditions can be obtained by the same procedure as the mode shapes of beams with general boundary conditions have been reported in [13].

\section{NUMERICAL SIMULATION AND DISCUSSIONS}

Numerical simulations of a clampedclamped beam with two cracks is presented in this section. Parameters of the beam are: Mass density $\rho=7800 \mathrm{~kg} / \mathrm{m}^{3}$; modulus of elasticity $E=2.0 \times 10^{11} \mathrm{~N} / \mathrm{m}^{2} ; L=1 \mathrm{~m} ; b=0.02 \mathrm{~m} ; h=$ $0.02 \mathrm{~m}$. Two cracks with the same depths are made at arbitrary positions of $0.4 \mathrm{~L}$ and $0.76 \mathrm{~L}$ from the left end of the beam. Five levels of the crack depth ranging from $0 \%$ to $20 \%$ have been applied. These five cases are numbered in Tab. 1. The first ten mode shapes are used to calculate the receptance and receptance curvature. The receptance and receptance curvature matrices are calculated at 100 points spaced equally on the beam while the force moves along these points. The Dirac delta function is approximated by the following formula [17]

$$
\delta(\xi)=\left\{\begin{array}{l}
0 \xi>\frac{\Delta \xi}{2} \\
\frac{1}{\Delta \xi}-\frac{\Delta \xi}{2} \leq x \leq \frac{\Delta \xi}{2} \\
0 \xi<-\frac{\Delta \xi}{2}
\end{array}\right.
$$

The value of the damage parameter $\lambda_{i}$ is determined as follows [12]

$$
\lambda_{i}=\frac{h}{L} C(\beta)
$$

where $C(\beta)=\frac{\beta(2-\beta)}{0.9(\beta-1)}$ and $\beta=\frac{d}{h}$ with $d$ is the crack depth. of varying depths

\begin{tabular}{cc}
\hline Case & Crack depth $(\%)$ \\
\hline 1 & 0 \\
2 & 10 \\
3 & 20 \\
4 & 30 \\
\hline
\end{tabular}




\subsection{Receptance of beam}

In our simulation the forcing frequency of the undamped beam should not be equal to the natural frequencies as the receptance will be infinitive, therefore we just investigate the receptance at the forcing frequency which is close to the natural frequencies. The difference between the forcing frequency and the natural frequency is $\Delta \omega=0.01 \mathrm{Rad} / \mathrm{s}$. Fig. 1 presents the 3D graphs of normalized receptance matrices at two forcing frequencies close to the first two natural frequencies of the intact beam. As can be seen from Fig. 1(a), when the forcing frequency is close to the first natural frequency, the receptance increases and reaches the maximum when the response point moves from the ends to the middle of beam. When the forcing frequency is close to the second natural frequency, the receptance is minimum at the middle of the beam and becomes maximum at positions $L / 4$ and $3 L / 4$ as depicted in Fig. 1(b). In order to show these receptance matrices in more details, the receptance along the beam when the force acts at a fixed point is extracted from these receptance matrices. Fig. 2 presents the receptance curves when the force acts at $0.42 \mathrm{~L}$. As can be observed from this figure, the receptance curves are smooth except the minimum position. These results imply that the responses of the beam can be estimated by using the receptance matrices when the forcing frequency is close to natural frequencies.

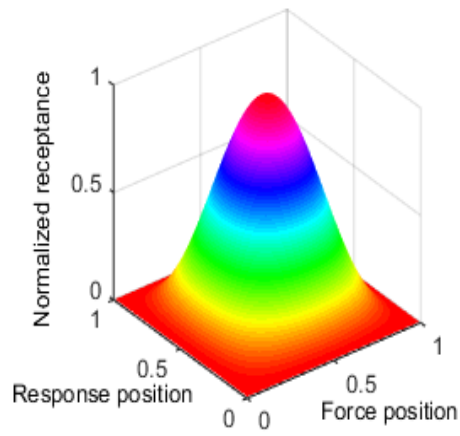

(a) $\omega \approx \omega_{1}$

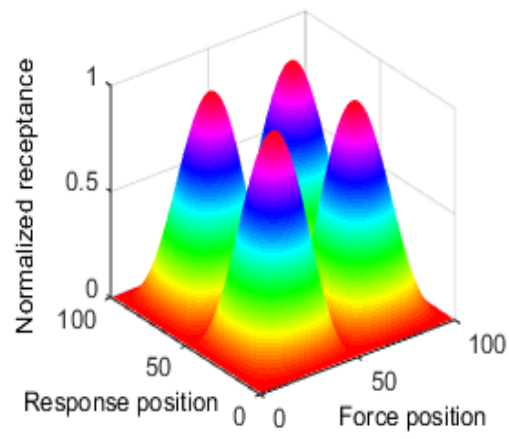

(b) $\omega \approx \omega_{2}$

Fig. 1. Receptance matrices of the intact beam

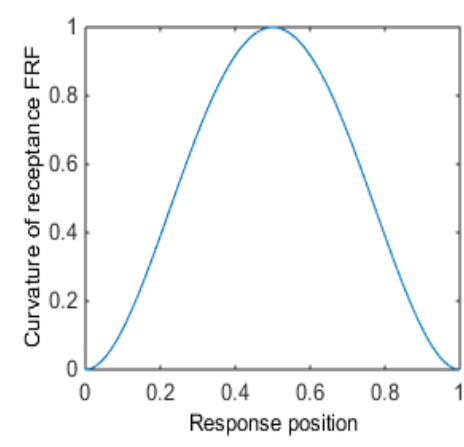

(a) $\omega \approx \omega_{1}$

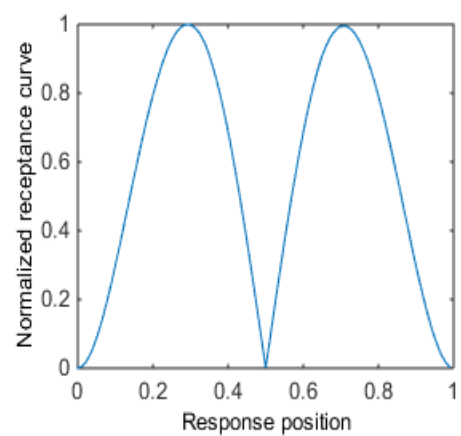

(b) $\omega \approx \omega_{2}$

Fig. 2. Receptance of the intact beam, $\hat{x}=0.4 L$ 
When there are cracks the mode shapes will have sharp changes at the crack positions leading to the changes in the receptance at the crack positions. Simulation results show that the changes in the receptance are small when the crack depth is small and they only become significant when the crack depth is large as depicted in Fig. 3. The distortions of the receptance matrix at the first natural frequency can only be observed when the crack depth is up to $50 \%$ of the beam height. As can be observed from Fig. 3(b) the distorted positions coincide with the crack positions at $0.34 \mathrm{~L}$ and $0.65 \mathrm{~L}$.

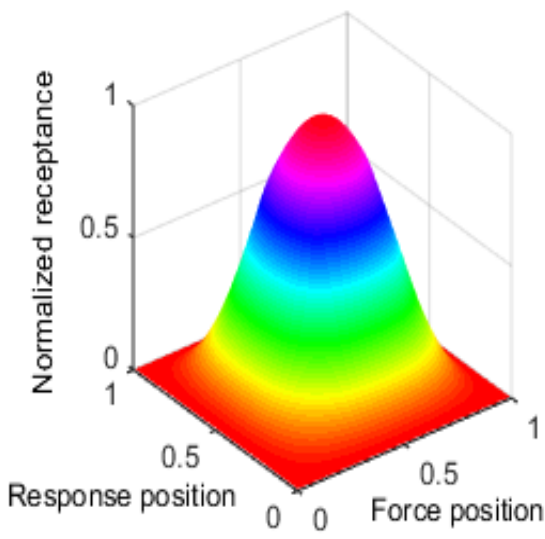

(a) Receptance matrix

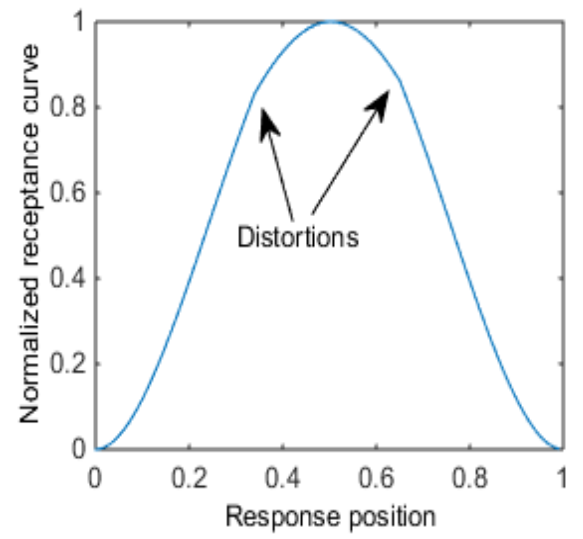

(b) Receptance with $\hat{x}=0.4 L$

Fig. 3. Receptance of the cracked beam, crack depth $=50 \%, \omega \approx \omega_{1}$

\subsection{Receptance curvature of the cracked beam}

When the crack depth is small, the receptance matrices have small changes at the crack positions, however, the receptance curvature matrices might have significant changes at the crack positions. Thus, in order $t 0$ investigate the influence of the small crack on the response, the receptance curvature of the cracked beam are applied. In this section, the receptance curvature matrices of the cracked beam with the crack depths of $10 \%$ and $20 \%$ are investigated. Figs. 4 and 5 depict the normalized receptance curvatures of the cracked beam with different levels of the crack depth when the forcing frequencies are close to the first and the second natural frequencies, respectively. As can be seen from these figures, there are sharp changes in the receptance curvature at crack positions. In order to determine exactly the sharp peak positions, the receptance curvatures along the beam is extracted when the force acts at a fixed position. The positions of these sharp changes can be inspected clearly as shown in Figs. 6 and 7 when the force acts at the position of $0.42 \mathrm{~L}$. As can be seen from these figures, the positions of the sharp changes are at $0.34 \mathrm{~L}$ and $0.65 \mathrm{~L}$ which coincide the crack positions. It should be noted from that for each level of the crack depth, the heights of sharp peaks are greater when the crack position is closer to the maxima of the receptance curvatures. Meanwhile, the heights of sharp peaks in receptance curvature are smaller when the crack position is far from the maxima of the receptance curvatures. 
These results mean that, the sharp peaks in receptance curvatures can be used for crack detection. In order to detect the cracks, only one receptance curvature measured along the beam when the force acts at a fixed point is needed. The sharp peaks in this measured receptance curvature indicate the existence of cracks, the positions of these sharp peaks point out the positions of cracks, and the heights of sharp peaks correspond to the crack severities. In addition, the numerical simulations show that in order to have better results for crack detection purpose, the force and the response should be applied at maximum positions of the receptance curvature matrices. When the crack is located at the minimum position of the receptance curvature, the crack cannot be detected. Clearly, only the receptance curvature corresponding to the first mode shape can be used for detecting arbitrary cracks on the beam since the first mode shape do not have any node.

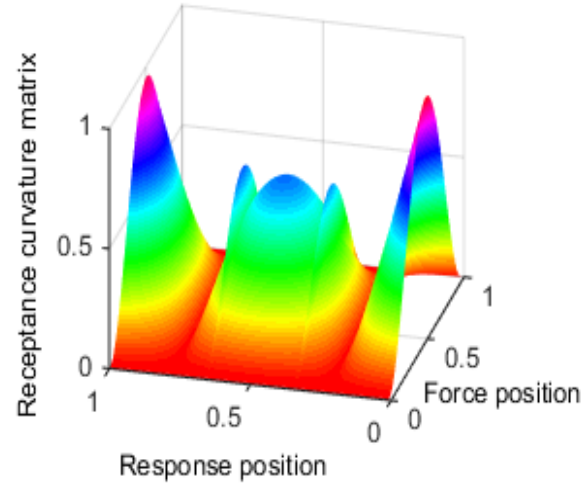

(a) $\omega \approx \omega_{1}$

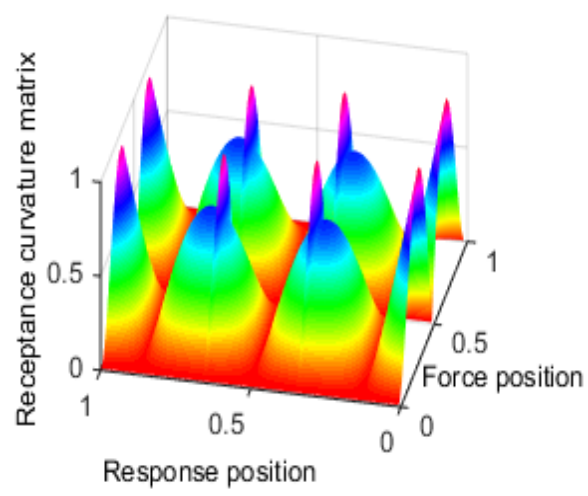

(b) $\omega \approx \omega_{2}$

Fig. 4. Receptance curvature matrices of the cracked beam, crack depth $=10 \%$

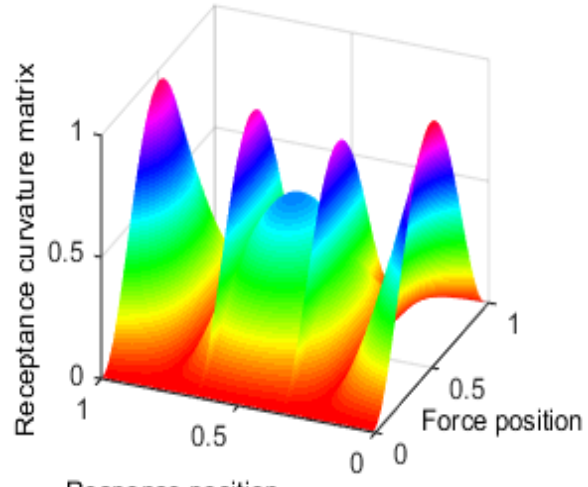

Response position

(a) $\omega \approx \omega_{1}$

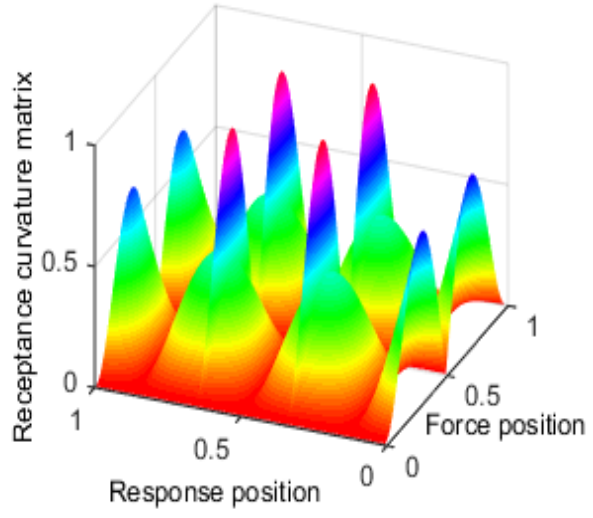

(b) $\omega \approx \omega_{2}$

Fig. 5. Receptance curvature matrices of the cracked beam, crack depth $=20 \%$ 


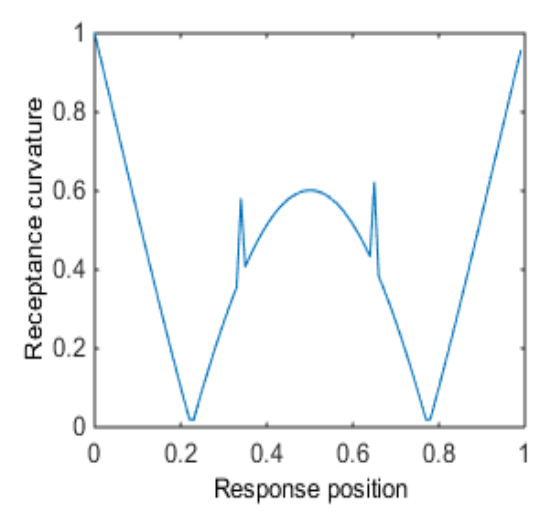

(a) $\omega \approx \omega_{1}$

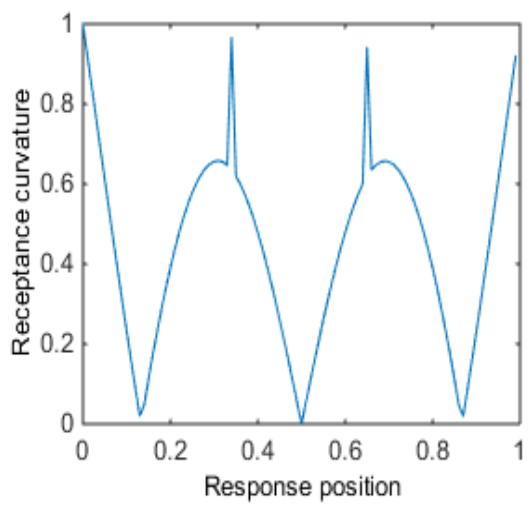

(b) $\omega \approx \omega_{2}$

Fig. 6. Receptance curvatures of the cracked beam, crack depth $=10 \%$

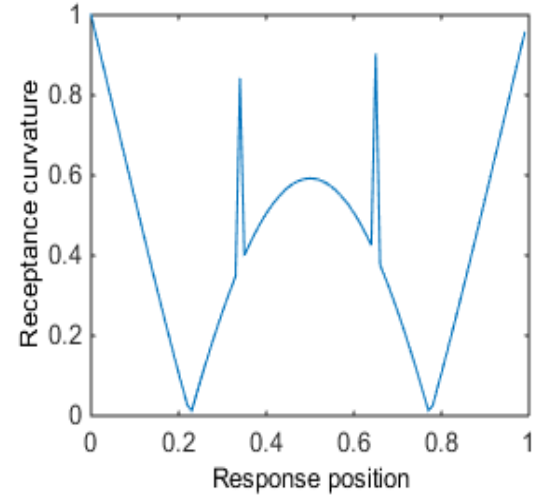

(a) $\omega \approx \omega_{1}$

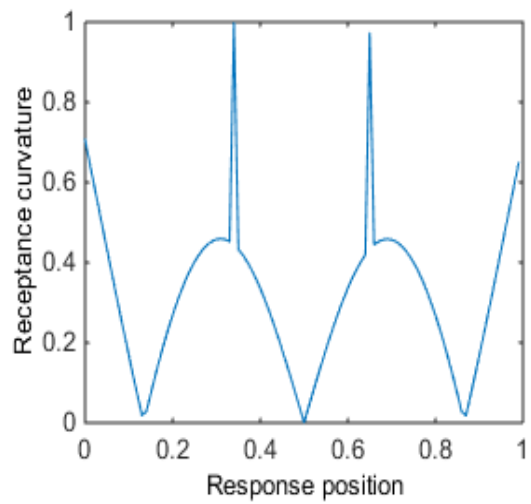

(b) $\omega \approx \omega_{2}$

Fig. 7. Receptance curvatures of the cracked beam, crack depth $=20 \%$

\section{Influence of the noise}

In order to simulate the polluted measurements, white noise is added to the receptance curvature obtained at the first frequency of the beam having two cracks at $0.34 \mathrm{~L}$ and $0.65 \mathrm{~L}$ with depths of $10 \%$. The white noise vector is obtained as following formula [18]

$$
\text { noise }=\sqrt{\frac{\sigma^{2}}{\exp \left(\frac{S N R * \ln (10)}{10}\right)}} * \mathbf{R},
$$

where $\sigma^{2}$ is the variance of the receptance curvature, SNR is the desired signal to noise ratio and $\mathbf{R}$ is a standard normal distribution vector with zero mean value and unit standard deviation. The noisy receptance curvature is the sum of the simulated receptance curvature and the noise vector presented in Eq. (23). 
Fig. 8 presents the noisy receptance curvatures at the first frequency with the SNR of 30 and 10. When the noise level SNR is of 40 , the two peaks at the crack positions can be inspected clearly as presented in Fig. 8(a). When the SNR equal to 20, the peaks at crack positions are presented but they are more difficult to be detected as shown in Fig. 8(b). These results suggest that, the proposed method can be applied efficiently to detect small crack depth from noisy measurements.

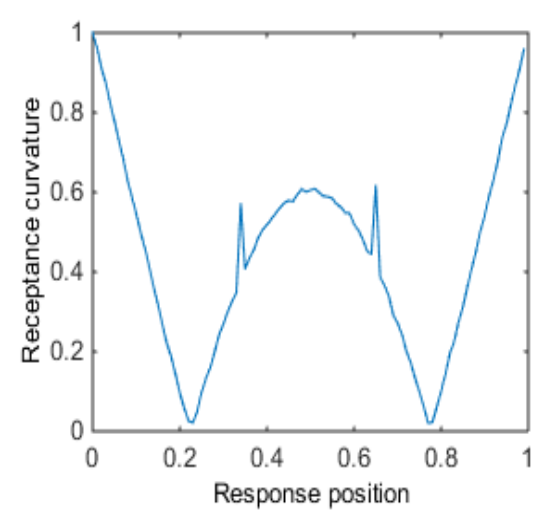

(a) $S N R=40$

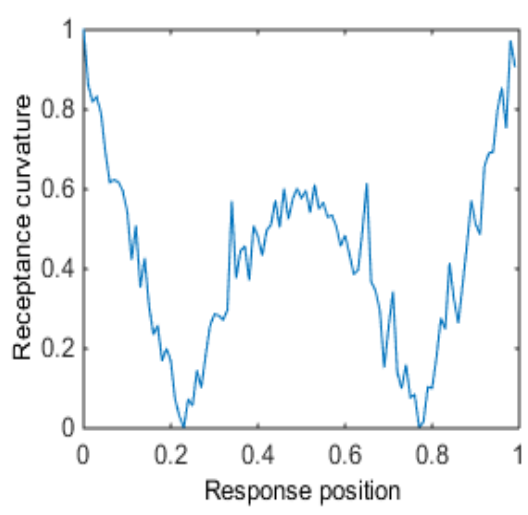

(b) $S N R=30$

Fig. 8. Receptance curvatures at the first natural frequency, crack depth $=10 \%, \hat{x}=0.42 \mathrm{~L}$

\section{CONCLUSIONS}

In this paper, exact formula of the receptance of a clamped-clamped cracked beam is presented. The advantage of the exact receptance function is that the frequency response at any point of a cracked beam can be easily predicted while the present receptance matrix methods can only predict the response at specific points.

The exact receptance curvature functions of cracked beams are also derived. When there are cracks, the receptance curvatures of the beam are changed significantly at the crack positions. The sharp peaks in the receptance curvatures can be detected clearly when the crack depth is as small as $10 \%$ of the beam height. This is an advantage of using the receptance curvature for early crack detection. The sharp peaks in the receptance curvature is the indicator of the existence of cracks and the positions of the sharp peaks show the positions of the cracks. The crack can be estimated by using noisy measurements of the receptance curvatures.

\section{ACKNOWLEDGEMENTS}

This research is funded by Vietnam National Foundation for Science and Technology Development (NAFOSTED) under grant number 107.02-2017.300.

\section{REFERENCES}

[1] R. E. D. Bishop and D. C. Johnson. The mechanics of vibration. Cambridge University Press, (1960). 
[2] B. Yang. Exact receptances of nonproportionally damped dynamic systems. Journal of Vibration and Acoustics, 115, (1), (1993), pp. 47-52.

[3] J. E. Mottershead. On the zeros of structural frequency response functions and their sensitivities. Mechanical Systems and Signal Processing, 12, (5), (1998), pp. 591-597. https://doi.org/10.1006/mssp.1998.0167.

[4] M. Gurgoze. Receptance matrices of viscously damped systems subject to several constraint equations. Journal of Sound and Vibration, 230, (5), (2000), pp. 1185-1190.

[5] A. Karakas and M. Gürgöze. A novel formulation of the receptance matrix of nonproportionally damped dynamic systems. Journal of Sound and Vibration, 264, (2003), pp. 733740. https://doi.org/10.1016/S0022-460X(02)01507-9.

[6] P. Albertelli, M. Goletti, and M. Monno. A new receptance coupling substructure analysis methodology to improve chatter free cutting conditions prediction. International Journal of Machine Tools and Manufacture, 72, (2013), pp. 16-24. https://doi.org/10.1016/j.ijmachtools.2013.05.003.

[7] G. Muscolino and R. Santoro. Explicit frequency response function of beams with crack of uncertain depth. Procedia Engineering, 199, (2017), pp. 1128-1133. https://doi.org/10.1016/j.proeng.2017.09.239.

[8] Y.-S. Lee and M.-J. Chung. A study on crack detection using eigenfrequency test data. Computers \& Structures, 77, (3), (2000), pp. 327-342. https://doi.org/10.1016/S0045-7949(99)001947.

[9] D. Y. Zheng and N. J. Kessissoglou. Free vibration analysis of a cracked beam by finite element method. Journal of Sound and Vibration, 273, (3), (2004), pp. 457-475. https://doi.org/10.1016/S0022-460X(03)00504-2.

[10] P. Gudmundson. The dynamic behaviour of slender structures with cross-sectional cracks. Journal of the Mechanics and Physics of Solids, 31, (4), (1983), pp. 329-345. https://doi.org/10.1016/0022-5096(83)90003-0.

[11] J. Thalapil and S. K. Maiti. Detection of longitudinal cracks in long and short beams using changes in natural frequencies. International Journal of Mechanical Sciences, 83, (2014), pp. 3847. https://doi.org/10.1016/j.ijmecsci.2014.03.022.

[12] N. V. Khoa. Monitoring a sudden crack of beam-like bridge during earthquake excitation. Vietnam Journal of Mechanics, 35, (3), (2013), pp. 189-202. https://doi.org/10.15625/0866$7136 / 35 / 3 / 2561$.

[13] S. Caddemi and I. Calio. Exact closed-form solution for the vibration modes of the EulerBernoulli beam with multiple open cracks. Journal of Sound and Vibration, 327, (3-5), (2009), pp. 473-489. https://doi.org/10.1016/j.jsv.2009.07.008.

[14] S. Caddemi and I. Caliò. Exact reconstruction of multiple concentrated damages on beams. Acta Mechanica, 225, (11), (2014), pp. 3137-3156. https://doi.org/10.1007/s00707-014-1105-5.

[15] T. V. Lien, N. T. Duc, and N. T. Khiem. Mode shape analysis of multiple cracked functionally graded beam-like structures by using dynamic stiffness method. Vietnam Journal of Mechanics, 39, (3), (2017), pp. 215-228. https://doi.org/10.15625/0866-7136/8631.

[16] K. V. Nguyen. Mode shapes analysis of a cracked beam and its application for crack detection. Journal of Sound and Vibration, 333, (3), (2014), pp. 848-872. https://doi.org/10.1016/j.jsv.2013.10.006.

[17] R. F. Hoskins. Delta function. Horwood Publishing Limited, second edition, (2009).

[18] R. Lyons. Understanding digital signal processing. Prentice Hall, Boston, USA, third edition, (2011). 